\title{
Dynamics of capillary spreading along hydrophilic microstripes
}

\author{
Anton A. Darhuber and Sandra M. Troian* \\ Microfluid Dynamics Laboratory, Department of Chemical Engineering, Princeton University, Princeton, New Jersey 08544 \\ Walter W. Reisner \\ Department of Physics, Princeton University, Princeton, New Jersey 08544 \\ (received 16 January 2001; published 13 August 2001)
}

\begin{abstract}
We have studied the capillary spreading of a Newtonian liquid along hydrophilic microstripes that are chemically defined on a hydrophobic substrate. The front of the spreading film advances in time according to a power law $x=B t^{1 / 2}$. This exponent of $1 / 2$ is much larger than the value $1 / 10$ observed in the axisymmetric spreading of a wetting droplet. It is identical to the exponent found for wicking in open or closed microchannels. Even though no wicking occurs in our system, the influence of surface curvature induced by the lateral confinement of the liquid stripe also leads to an exponent of $1 / 2$ but with a strongly modified prefactor $B$. We obtain excellent experimental agreement with the predicted time dependence of the front location and the dependence of the front speed on the stripe width. Additional experiments and simulations reveal the influence of the reservoir volume, liquid material parameters, edge roughness, and nonwetting defects. These results are relevant to liquid dosing applications or microfluidic delivery systems based on free-surface flow.
\end{abstract}

DOI: 10.1103/PhysRevE.64.031603

PACS number(s): 68.08.Bc, 47.85.Np, 68.15.+e, 68.03.Cd

\section{INTRODUCTION}

The kinetics of capillary phenomena such as spreading, wetting, wicking, and imbibition control the quality of liquid film deposition in numerous applications like coating, painting, printing, and lubrication. Several decades of work ranging from theoretical analysis and molecular dynamics simulations to time resolved ellipsometric studies have elucidated how intermolecular forces control the spreading and flow of thin films [1-3]. Nowadays, the study of capillary phenomena and microfluid dynamics is regaining attention as technological advances facilitate the miniaturization of devices for chemical analysis or medical diagnostics. The chemical patterning of substrates or closed channels into regions of mixed wettability has introduced interesting approaches for fluid migration and flow control in confining networks.

The capillary spreading of a liquid droplet on a smooth and chemically homogeneous surface has been investigated extensively. Tanner [4] first measured the radial advance of a completely wetting droplet of a Newtonian liquid on a smooth substrate and found $r(t) \sim\left(\gamma / \eta \Omega^{3} t\right)^{1 / 10}$ where $\gamma$ is the liquid surface tension, $\eta$ the liquid viscosity, and $\Omega$ the droplet volume. He also confirmed this result with a quasistatic hydrodynamic model. Later studies revealed that for completely wetting liquids, the contact line is preceded by a thin precursor film of molecular dimensions [5-7] whose evolution follows diffusion dynamics according to $K t^{1 / 2}$. The prefactor $K$ was found to depend on the liquid's molecular weight and the relative humidity [7].

The spreading rate of liquids on corrugated surfaces can be enhanced by capillary migration and wicking into narrow surface grooves. Various experimental and theoretical studies have explored liquid advances on a surface with random or

\footnotetext{
*Author to whom correspondence should be addressed. Electronic address: stroian@princeton.edu
}

periodic grooves both parallel and perpendicular to the direction of spreading [8-18]. These studies have shown that completely wetting liquids spreading along streamwise oriented grooves follow Washburn-type dynamics, [20] wherein the liquid front advances as $t^{1 / 2}$. Hydrodynamic modeling of wicking into a surface groove of arbitrary and constant cross section confirms this exponent and reveals that the front speed as a function of distance is proportional to the groove depth $[12,15]$.

In this paper we consider the spreading of a Newtonian liquid on smooth and flat but chemically micropatterned surfaces. The patterns consist of narrow hydrophilic stripes on a hydrophobic background. The liquid is confined to these hydrophilic areas and does not spread onto the hydrophobic regions. Our experiments show that the liquid front advances in time as $t^{1 / 2}$ despite the fact that no capillary wicking occurs. Moreover, the front speed as a function of position is found to depend on the fourth power of the stripe width. This is a significantly stronger size dependence than observed for liquids spreading in capillary tubes or along surface grooves. We develop a hydrodynamic lubrication model, which shows that the dynamics are dominated by the transverse curvature of the air-liquid interface. The results derived for the spreading exponent and the dependence on stripe width and liquid volume are in excellent agreement with experiment.

\section{EXPERIMENTAL SETUP}

The substrates used in the spreading experiments were fabricated from glass slides (Corning $1737 \mathrm{~F}$ ) and silicon wafers [(001) oriented, $n$-type doped with resistivity 10-20 $\Omega \mathrm{cm}]$. The samples were cleaned with acetone and isopropanol and then immersed in a mixture of sulfuric acid, hydrogen peroxide, and deionized water at $80^{\circ} \mathrm{C}$ for $15 \mathrm{~min}$. This step was followed by 10 min of oxygen plasma cleaning. The silicon wafers were further treated by immersion into concentrated hydrofluoric acid for $10 \mathrm{~min}$. The samples 


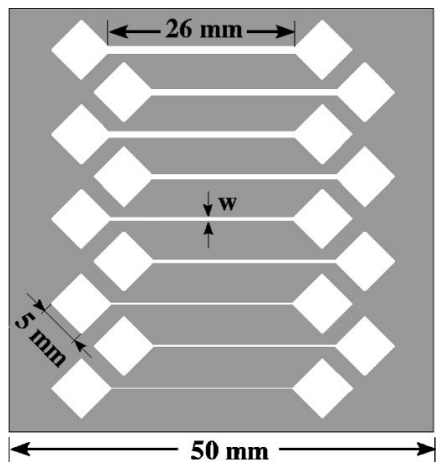

FIG. 1. Diagram of the sample layout. The width of the square reservoir pads is $5 \mathrm{~mm}$; the length of the stripes connecting the reservoirs is $26 \mathrm{~mm}$.

were coated with a self-assembled monolayer of $1 \mathrm{H}-1 \mathrm{H}-2 \mathrm{H}-$ 2H-Perfluorooctyl-trichlorosilane (PFOTS, Fluka) [21,22].

The hydrophilic patterns were defined with photolithography and reactive ion etching in a Plasmatherm 790 using oxygen plasma for $12 \mathrm{~min}\left(\mathrm{O}_{2}\right.$ flow rate $39 \mathrm{SCCM}$, pressure 100 mTorr, power 40-70 W). Since the thickness of the monomolecular PFOTS coating is only about $2 \mathrm{~nm}$, the substrate presents a practically flat surface to the oncoming liquid flow. The masks used for photolithography were printed with a commercial image setter. The stripe edges showed a roughness and waviness of amplitude on the order of $5 \mu \mathrm{m}$, which is far smaller than the stripe width. The hydrophilic patterns consist of square-shaped reservoir pads $5 \mathrm{~mm}$ in width, which are connected by $26-\mathrm{mm}$ long hydrophilic stripes. The stripe width $w$ ranged from 100 to $800 \mu \mathrm{m}$. The sample layout is depicted in Fig. 1.

The liquid used in this study was polydimethylsiloxane silicone oil DC200 (Fluka) of viscosity $\mu=20 \mathrm{mPas}$. The surface tension $\gamma$ is $20.6 \mathrm{mN} / \mathrm{m}$ and the density $\rho$ is 954 $\mathrm{kg} / \mathrm{m}^{3}$. Liquid droplets were deposited on the reservoir pad with a Hamilton digital syringe with a volume resolution of $0.1 \mu l$. The spreading front was tracked with an Olympus BX-60 microscope equipped with a CCD camera and a green passband filter centered about $537 \mathrm{~nm}$. The leading edge of an advancing rivulet was imaged via optical fringes resulting from interference between the air-liquid interface and the solid substrate. To maintain isothermal conditions, the substrates rested on a solid metal block and the light source was equipped with an infrared cutoff filter.

\section{THEORETICAL DESCRIPTION}

\section{A. Horizontal capillaries-the Washburn equation}

For a Newtonian liquid wicked into a small circular capillary of radius $R$, the meniscus shape closely approximates a spherical cap of radius $R / \cos \theta$, where $\theta$ represents the advancing contact angle assumed constant. The meniscus curvature lowers the pressure in the liquid phase by $\Delta p$ $=2 \gamma \cos \theta / R$. There exists a pressure difference, therefore, between the inlet and the meniscus front given by $2 \gamma \cos \theta / R$. The balance of this wicking pressure against the viscous drag on the wall produces a radially averaged veloc- ity profile given by $\langle u\rangle=\left(R^{2} / 8 \mu\right)(\Delta p / x)$, where $x$ represents the advancing meniscus position along the capillary. The differential equation $\langle u\rangle=d x / d t=(R \gamma \cos \theta / 4 \mu)(1 / x)$ results in the well known Washburn equation [20]

$$
x-x_{0}=\left(\frac{R \gamma \cos \theta}{2 \mu}\right)^{1 / 2}\left(t-t_{0}\right)^{1 / 2},
$$

where $x_{0}$ is the initial position of the moving air-liquid interface and $t_{0}$ denotes the initial time. The characteristic $t^{1 / 2}$ scaling reflects the fact that a constant pressure difference $\Delta p$ is exerted over an ever increasing length $x$. This scaling relation holds equally well for capillaries of constant but noncircular cross section with modification only to the prefactor in Eq. (1). Krotov and Rusanov [23] studied the wicking speed for capillaries of circular, square, and triangular shape and found that $\langle u\rangle$ was largest for tubes with triangular cross-section.

Equation (1) predicts that the liquid front speed $\langle u\rangle$ is proportional to the capillary tube radius and inversely proportional to the distance traveled. Deviations from the Washburn equation, especially at early times, have been observed by Joos et al. in capillary rise experiments [24]. The deviations have been attributed to inertial effects for capillary radii larger than about $500 \mu \mathrm{m}$ and to the velocity dependence of the advancing contact angle. Bubble formation in water filled capillaries of radii $r<0.3 \mu \mathrm{m}$ has also been found to cause deviations from the Washburn equation [25].

\section{B. Liquid spreading in surface grooves}

Summ et al. [8] and Rye et al. [13] studied the wicking process into open surface grooves and found that the meniscus advanced according to $x=K^{\prime}(\theta, \alpha) \sqrt{\gamma d / \mu} t^{1 / 2}$, where $d$ is the depth of the surface groove and $K^{\prime}(\theta, \alpha)$ a geometric factor, which depends on the advancing contact angle $\theta$ and the apex angle $\alpha$ of the groove. Despite the nonenclosed geometry and the free-surface flow, the $t^{1 / 2}$ behavior and the linear dependence on the characteristic dimension persist.

This result may seem surprising at first, since the flow field in a closed capillary differs significantly from the velocity profile of liquid in a surface groove that maintains a free and deformable surface. However, the $t^{1 / 2}$ kinetics are robust so long as the pressure difference $\Delta p$ driving the flow is independent of the meniscus position. Deviations to the $t^{1 / 2}$ scaling, especially for early times, can result from a variation of the advancing contact angle with the speed of the wetting line, from changes in the groove geometry and from inertial and streamwise surface curvature terms, which decay quickly as $t$ increases [14].

\section{Capillary spreading along hydrophilic stripes}

In the following we consider the capillary spreading of a Newtonian liquid confined to flow on a hydrophilic microstripe residing on a hydrophobic background. Referring to Fig. 1, a small liquid volume is deposited onto one of the square-shaped hydrophilic reservoir pads that are connected to long and narrow hydrophilic stripes. The liquid spontane- 


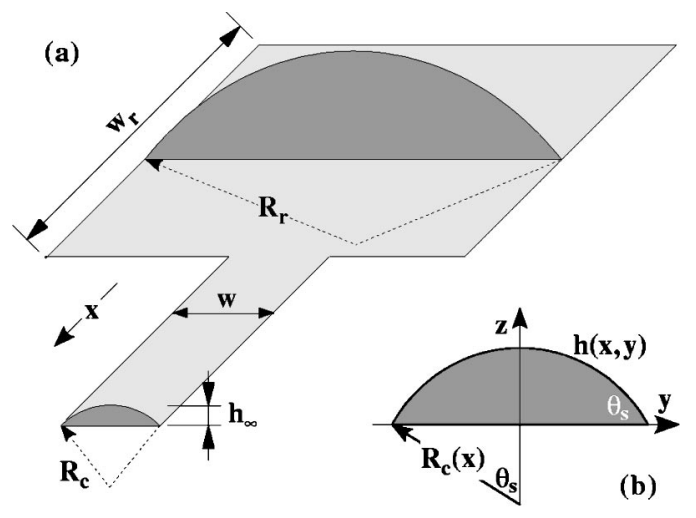

FIG. 2. (a) Coordinate system and nomenclature used in the description of a liquid sample deposited on a square reservoir pad and spreading along a hydrophilic stripe. (b) The transverse crosssectional profile of a liquid rivulet is a segment of a circle. $\theta_{s}$ denotes the apparent contact angle in a plane perpendicular to the hydrophilic/hydrophobic boundary line.

ously flows from the reservoir along the microstripe thereby relaxing the advancing contact angle toward its static equilibrium value $\theta_{\mathrm{eq}}$. In our case $\theta_{\mathrm{eq}}$ is zero, such that the liquid will continue to spread until it covers the entire hydrophilic surface area. Hosking et al. have also studied capillary spreading in a similar geometry of comparable size. However, they considered the more complex problem of a multicomponent liquid solder reacting with the copper surface during spreading [19].

We first discuss the asymptotic solution for the liquid surface profile that describes the final equilibrium configuration. We then derive the equation governing the dynamic evolution of the liquid stripe and solve it using a self-similarity transformation.

\section{Asymptotic solution}

A liquid droplet deposited on a reservoir pad will adopt a surface with mean curvature $2 / R_{r}$, where $R_{r}$ is its radius of curvature. If the stripe width is much smaller than the reservoir size, drainage into the stripe can be neglected, and $R_{r}$ remains essentially constant in time. In the asymptotic limit, the system maintains a constant capillary pressure throughout the liquid. Consequently, the cross sectional profile in the channel is a sector of a circle with apex height $h_{\infty}$. Moreover, the radius of curvature $R_{c}$ is constant along the hydrophilic channel [see Fig. 2(a)]. Assuming gravitational effects to be negligible, the condition of constant mean curvature further requires that $2 / R_{r}=1 / R_{c}$. For small values of $\theta_{s}$ [see Fig. 2(b)], $h_{\infty}=R_{c}\left(1-\cos \theta_{s}\right) \approx R_{c} \theta_{s}^{2} / 2$ and $w=2 R_{c} \sin \theta_{s}$ $\approx 2 R_{c} \theta_{s}$, which results in the relation

$$
h_{\infty}=w^{2} / 8 R_{c}=w^{2} / 4 R_{r} .
$$

In our experiments, the width of the reservoir pads is 5 $\mathrm{mm}$, which is larger than the capillary length for silicone oil $l_{c}=\sqrt{\gamma / \rho g} \approx 1.5 \mathrm{~mm}$. Hence, the condition of constant pressure is actually not equivalent to the condition of constant curvature. For length scales exceeding $l_{c}$, the gravitational pressure must be taken into account. In this case, the relation
$2 / R_{r}=1 / R_{c}$ is replaced by $p(0,0)=\gamma / R_{c}$, where $p(0,0)$ is the sum of the capillary and hydrostatic pressure at the bottom of the reservoir pad. This relation clearly does not affect the square dependence of $h_{\infty}$ on the stripewidth [Eq. (2)] as also verified by numerical simulations using SURFACE EVOLVER [26].

\section{Evolution equation}

In what follows, we consider flow along microstripes for which the Bond number $\mathrm{Bo}=\rho g w^{2} / \gamma \ll 1$ and the capillary number $\mathrm{Ca}=\mu\langle u\rangle / \gamma \ll 1$. In addition, the stripe width $w$ is much smaller than the reservoir size and consequently, the liquid height on the stripe is much smaller than $w$. For well developed flow such that the distance spread, $L$, is much longer than the stripe width $w$, the transverse and vertical components of the velocity, $u_{y}$ and $u_{z}$, are negligible. In this lubrication approximation, [27] volume conservation determines the evolution of the liquid cross-sectional area $A$ according to

$$
\frac{\partial A}{\partial t}+\frac{\partial Q}{\partial x}=0
$$

where $\partial Q / \partial x$ is the longitudinal gradient of the volumetric flow rate $Q$. For liquids with low vapor pressure under isothermal conditions, the mechanism driving spreading is the longitudinal capillary pressure gradient $\partial p / \partial x$ where $p \approx$ $-\gamma\left(\partial^{2} h / \partial x^{2}+\partial^{2} h / \partial y^{2}\right)$. Since the longitudinal curvature term is negligible for $w / L \ll 1, \partial p / \partial x \approx-\gamma \partial^{3} h / \partial x \partial y^{2}$. In the limit $\mathrm{Ca} \ll 1$, there is no viscous contribution to the capillary pressure. In addition, the lubrication regime requires that $u_{y} \sim \partial p / \partial y=0$. Consequently, the transverse crosssectional profile of the liquid spreading along the hydrophilic stripe is a curve of constant curvature, i.e., an arc of a circle. The cross-sectional area $A$ is therefore given by $A(x)$ $=2 \int_{0}^{w / 2} h(x, y) d y$, where $h(x, y)=h_{c}(x)\left(1-4 y^{2} / w^{2}\right)$ and $h_{c}(x)$ is the local apex height of the liquid rivulet. The boundary conditions of no slip at the stripe surface $\left[u_{x}(z\right.$ $=0)=0]$ and vanishing shear stress at the air-liquid interface $\left[\partial u_{x} / \partial z(z=h)=0\right]$ lead to a Poiseuille-like velocity profile

$$
u_{x}=\frac{1}{\mu} \frac{\partial p}{\partial x}\left(\frac{z^{2}}{2}-h z\right)
$$

The volumetric flow rate is therefore given by

$$
Q=\int_{-w / 2}^{w / 2} \int_{0}^{h} \frac{1}{\mu} \frac{\partial p}{\partial x}\left(\frac{z^{2}}{2}-h z\right) d z d y
$$

In analogy to the derivation of Eq. (2), the dominant contribution to the pressure gradient is given by

$$
\frac{\partial p}{\partial x} \approx-\gamma \frac{\partial^{3} h}{\partial x \partial y^{2}}=\frac{\partial}{\partial x}\left(\frac{\gamma}{R_{c}}\right) \approx \frac{8 \gamma}{w^{2}} \frac{\partial h_{c}}{\partial x} .
$$

Substitution into Eq. (3) yields the evolution equation for the apex height $h_{c}(x)$ 


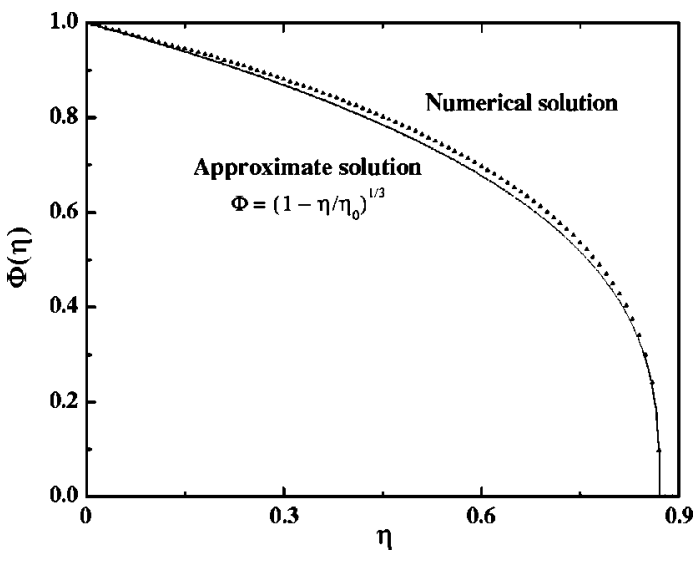

FIG. 3. Numerical (triangles) and analytical solution (continuous line) corresponding to Eq. (9).

$$
\frac{\partial h_{c}}{\partial t}-\frac{64}{35} \frac{\gamma}{\mu w^{2}} \frac{\partial}{\partial x}\left(h_{c}^{3} \frac{\partial h_{c}}{\partial x}\right)=0 .
$$

Strictly speaking, this equation does not hold at the reservoir inlet, where the assumption of a shallow profile $h \ll w$ is invalid. Moreover, it does not hold close to the advancing contact line, since the flow field at the front is not strictly unidirectional and the longitudinal curvature cannot be neglected. However, Eq. (7) is expected to describe the flow in between these limits properly.

\section{Self-similar solution for the spreading profile}

Following Ref. [15], a solution to Eq. (7) can be found by applying a self-similarity transformation $[28,29]$ with dimensionless variables

$$
\Phi=h / h_{\infty} \text { and } \eta=\frac{x}{\sqrt{D t}}
$$

where

$$
D=\frac{64}{35} \frac{\gamma h_{\infty}^{3}}{\mu w^{2}},
$$

$h_{\infty}$ represents the apex film height at the stripe inlet in the asymptotic limit. This change of variables transforms the partial differential equation (7) into an ordinary differential equation

$$
\frac{d}{d \eta}\left(\Phi^{3} \frac{d \Phi}{d \eta}\right)+\frac{\eta}{2} \frac{d \Phi}{d \eta}=0 .
$$

The boundary conditions are $\Phi(0)=1$ and $\int_{0}^{\infty} \Phi d \eta<\infty$. The second constraint, which is equivalent to the liquid volume being finite, requires that $\Phi(\eta)$ maintain a value of zero beyond some limiting distance $\eta_{0}$. The numerical solution shown in Fig. 3 yields the value $\eta_{0} \approx 0.87$. A close approximation to the exact numerical solution can be found by expanding $\Phi(\eta)$ in a power series and solving Eq. (9) to first order. A comparison between this approximate form $\Phi(\eta)$ $=\left(1-\eta / \eta_{0}\right)^{1 / 3}$ and the numerical solution is shown in Fig. 3. The slope and curvature of these solutions have a singularity at the position of the contact line $\eta_{0}$ that can be eliminated by consideration of a molecularly thin precursor film [1].

According to the similarity transformation in Eq. (8), the wetting front advances as $x=\sqrt{D t}$. This is the same time dependence as for a liquid wicking into a small capillary or surface groove. Again, the $t^{1 / 2}$ behavior reflects a constant pressure difference exerted over an increasing length $L$. The average streamwise velocity is therefore given by

$$
\langle u\rangle=\frac{d x}{d t} \sim \sqrt{\frac{D}{t}} \sim \frac{\gamma}{\mu} \frac{h_{\infty}^{3}}{w^{2}} \frac{1}{x} \sim \frac{\gamma w^{4}}{\mu} \frac{1}{x} .
$$

Thus, the spreading speed, $d x / d t \sim w^{4}$, exhibits a strong dependence on the stripe width. Equation (10) also determines the dependence of the velocity on the volume $V$ of liquid deposited on the reservoir pad. As pointed out in Sec. III C $1, h_{\infty}=h_{c}(x=0)$ is inversely proportional to $R_{r}$ for pad sizes $w_{r}$ smaller than the capillary length $l_{c}$. Since $V$ $\sim 1 / R_{r} \sim h_{\infty}$, we therefore expect $\langle u\rangle$ to be proportional to the third power of the volume $V^{3}$. For pad dimensions exceeding $l_{c}$, as in our case, gravity noticeably flattens the profile of the air-liquid interface above the reservoir pad. The dependence of the hydrostatic pressure at the bottom of the reservoir pad is slightly less than linear in the liquid volume. SURFACE EVOLVER calculations gave the result $p(0,0)$ $\sim V^{0.92}$ for a pad dimension of $5 \mathrm{~mm}$. Consequently, we expect a dependence $\langle u\rangle \sim V^{2.76}$.

\section{Surfaces of constant capillary pressure}

Once the liquid rivulet extends far into the channel, i.e., when $L \gg w$, the change in the capillary pressure $\Delta p$ is small on a lengthscale $\Delta x \approx w$. It is therefore a good approximation to regard the air-liquid interface locally as a surface of constant mean curvature. We have used the software package SURFACE EVOLVER [26] to study the influence of stripe edge roughness and nonwetting defects on the surface profile of liquids confined to narrow hydrophilic lines. Hydrostatic forces were included in all simulations, however, because of the small stripe width, their effect was negligible in comparison to the surface tension forces. The results of the simulations for $w=500 \mu \mathrm{m}$ presented below can therefore be directly scaled down to smaller feature sizes.

\section{Influence of stripe edge roughness}

Due to the no-slip boundary condition, the streamwise velocity vanishes at the edges of the chemically patterned stripe defining the boundary between the hydrophilic and the exterior hydrophobic region. Microscopic roughness of these edges is therefore not expected to influence the flow dynamics significantly. The effect of edge irregularities on the surface profile of a liquid rivulet depends strongly on the wavelength spectrum. Figure 4 shows the longitudinal variation of the apex height for a liquid rivulet on a hydrophilic stripe of average width $\langle w\rangle=500 \mu \mathrm{m}$ and with a sawtooth edge corrugation of amplitude $a=10 \mu \mathrm{m}$. When the corrugation 


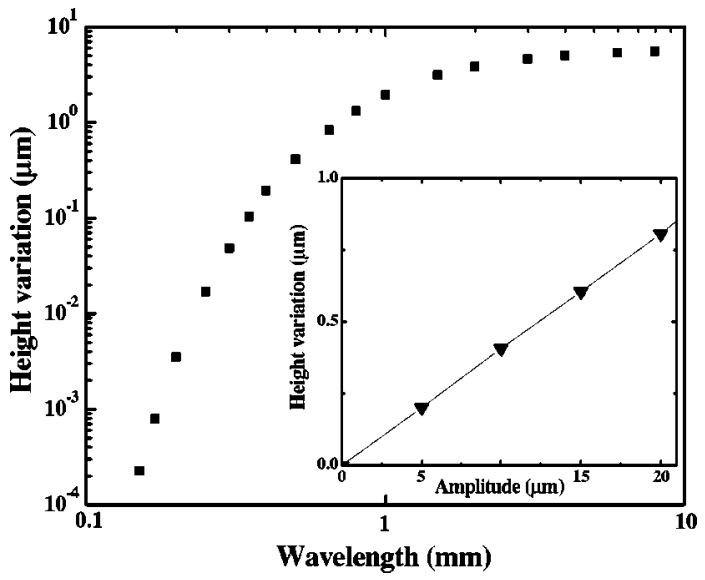

FIG. 4. Apex height variation of a static liquid rivulet confined to a corrugated hydrophilic stripe. The edge roughness has a sawtooth contour with longitudinal wavelength $\lambda$ and amplitude $a$ $=10 \mu \mathrm{m}$. The average width of the channel $\langle w\rangle$ is $500 \mu \mathrm{m}$; the average height is $h_{0}=50 \mu \mathrm{m}$. Inset: dependence of the height variation on the amplitude of the channel edge roughness for a wavelength $\lambda=\langle w\rangle$.

wavelength is much larger than the stripe width, the liquid height variation saturates at a value $h_{\max } / h_{\min }$ $=\left(w_{\max } / w_{\min }\right)^{2}$, provided the lateral liquid contact angle $\theta_{s}$ is small. However, as the corrugation wavelength decreases below the stripe width, a highly nonlinear decay of the height variation is observed. The dependence of the height variation on the roughness amplitude $a$ is linear for small values of $a /\langle w\rangle$ as shown in the inset of Fig. 4.

These results suggest that small amplitude edge roughness with a wavelength smaller than the average stripe width does not have any significant influence on the velocity or the surface profile of the spreading liquid. However, long range variations of the average channel width can manifest themselves in a noticeable modulation of the longitudinal height profile.

\section{Influence of nonwetting surface defects}

Contact line distortions due to the presence of surface heterogeneities and defects have been studied experimentally and theoretically by a number of groups [30-36]. Neglecting gravity, Shanahan [35] studied the spatial decay of a localized contact line distortion for the case of a droplet with small equilibrium contact angle $\theta_{0}$. In the limit of large droplet radii $r_{0}$, he derived the asymptotic form of the wetting line distortion $\delta(x) \sim f\left(\pi \gamma \theta_{0}^{2}\right)^{-1} \ln \left(r_{0} /|x|\right)$, where $x$ denotes the coordinate axis perpendicular to the radial direction and $f$ the localized force responsible for the distortion of the triple line. Joanny and de Gennes [30] had derived a similar result under slightly different approximations. According to this model, contact line distortions decay logarithmically on a length scale governed by the droplet radius. The prefactor of the logarithmic term is inversely proportional to $\theta_{0}^{2}$.

We performed energy minimization simulations to determine the surface profile of a $3 \mathrm{~mm}$ liquid ribbon confined to a hydrophilic microstripe containing a small nonwetting circular defect. Figure 5(a) shows the surface profile of a $500-\mu \mathrm{m}$ wide liquid ribbon with a $20-\mu \mathrm{m}$ diameter nonwetting defect located at the stripe center. The height profile decreases to zero at the perimeter of the defect. This reduces the effective apex height of the liquid rivulet to approximately one-half the undisturbed value far upstream or downstream of the defect. Figure 5(b) shows longitudinal crosssections for three different values of the undisturbed liquid height $h_{c}$. As can be seen, the decay length of the height profile is proportional to the width of the hydrophilic stripe but practically independent of $h_{c}$. This result cannot be directly compared to Shanahan's results since his model describes the distortion of a contact line while ours considers the decay length of the film height in the vicinity of a dewetted spot.

Figure 5(c) shows three sets of transverse cross sections for three different values of the rivulet height $h_{c}$. The dashed lines represent the unperturbed film heights far upstream of the defect. The solid lines show the liquid distortion in the vicinity of the defect. The contact angle at the periphery of the defect varies strongly with $h_{c}$, potentially leading to a flooding of the defect if $h_{c}$ exceeds a critical value. This critical value increases with the ratio of the defect size to the channel width and the value of the advancing contact angle on the defect. Figure 5(d) shows transverse cross-sections of the height profiles for defects displaced from the center towards the edge of the hydrophilic boundary. As the defect patch is shifted towards the edge, the apex height approaches the upstream value and the contact angle at the defect perimeter decreases. Defects that are located close to the stripe edges are therefore much less likely to be flooded than defects in the center of the channel.

It is expected that the presence of a small nonwetting defect on the spreading of a liquid rivulet along a hydrophilic lane should decrease the average height over a longitudinal interval $\Delta x \approx 2 w$, which in turn should increase the viscous drag in this region. Experimentally, we find that the spreading speed recovers its undisturbed value once the triple line has advanced a distance on the order of $2 w$ past the defect location.

\section{Front shapes of static liquid ribbons}

We calculated the surface profile and the contact line shape for liquid ribbons confined to a $500-\mu \mathrm{m}$ wide stripe for different values of the equilibrium contact angle $\theta_{e q}$. Figure 6(b) shows longitudinal cross sections of the height profiles for three values of $\theta_{e q}=5^{\circ}, 10^{\circ}$, and $20^{\circ}$. As in the case of nonwetting defects, the length scale over which the height profile decreases to zero at the contact line is equal to the stripe width irrespective of $\theta_{e q}$. If $\theta_{e q}$ is increased beyond these small values, the surface profile may develop bulges [37]. For small values of $\theta_{e q}$, however, there is a linear relation between the maximum height and the contact angle.

The liquid-solid contact lines on a $500-\mu \mathrm{m}$ wide hydrophilic stripe for three values of $\theta=10^{\circ}, 20^{\circ}$, and $30^{\circ}$ are shown in Fig. 6(a). Shown for comparison is a continuous line, which is a circular arc of radius $267 \mu \mathrm{m}$. Interestingly, the shape of the wetting line is independent of the value of 

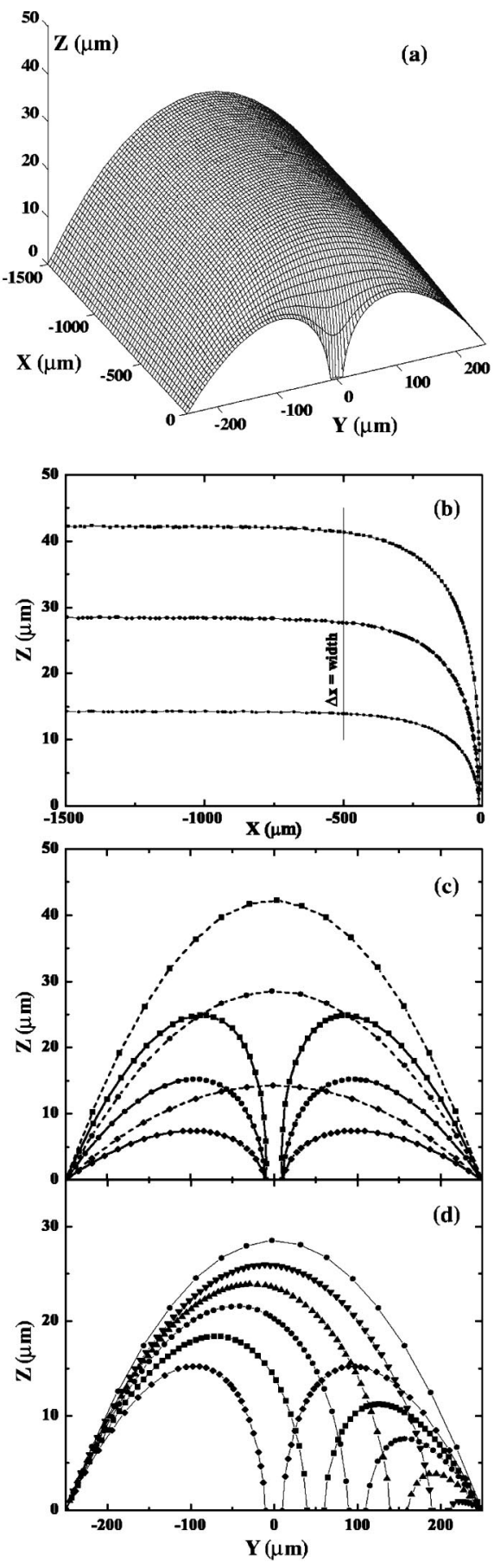

FIG. 5. Equilibrium surface profiles of a liquid rivulet in the presence of a defect placed within the hydrophilic stripe. (a) Profile of an infinitely long and $500 \mu \mathrm{m}$ wide liquid rivulet subject to a circular nonwetting defect of diameter $20 \mu \mathrm{m}$ centered at $(X, Y)$ $=(0,0)$. (b) Longitudinal sections of the surface profiles for three different values of the asymptotic liquid height $h_{c}$ subject to the same defect as in (a). The decay length of the height profile from $h_{c}$ to zero is proportional to the width of the channel and practically independent of $h_{c}$. (c) Transverse sections of the height profile for three different values of $h_{c}$. The dashed lines represent the unperturbed film heights far upstream of the defect. The solid lines show the liquid distortion in the vicinity of the defect. (d) Transverse sections of the height profile for defects displaced towards the stripe edge.

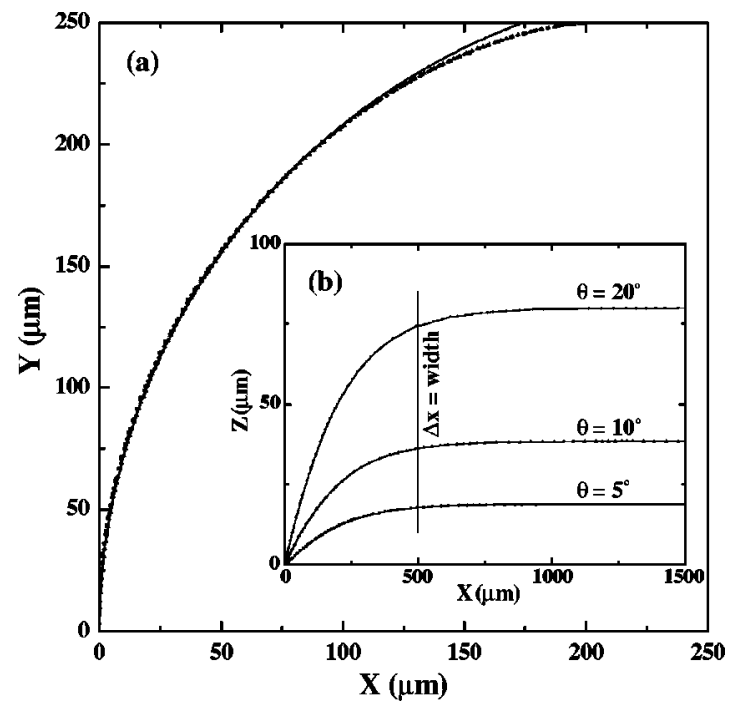

FIG. 6. (a) Equilibrium shape of the contact line for three different values of the contact angle $\theta_{e q}=10^{\circ}, 20^{\circ}$, and $30^{\circ}$. The continuous line is an arc of a circle with radius $267 \mu \mathrm{m}$. The stripe width is $500-\mu \mathrm{m}$. (b) Longitudinal sections of a long liquid droplet confined to a $500-\mu \mathrm{m}$ wide hydrophilic channel for three values of $\theta_{e q}=5^{\circ}, 10^{\circ}$, and $20^{\circ}$.

$\theta_{e q}$ and indistinguishable from a circular arc except at the very edge of the hydrophilic/hydrophobic boundary.

\section{EXPERIMENTAL RESULTS}

We have measured the front velocity of liquid rivulets spreading from reservoir pads onto hydrophilic stripes of widths ranging from 200 to $800 \mu \mathrm{m}$. The velocity as a function of position was extracted from image sequences recorded with a CCD camera at defined time intervals. Experimental data are shown in Fig. 7(a). For distances $x$ from the reservoir inlet larger than $5-10 \mathrm{~mm}$, the curves follow a power-law behavior $v \sim x^{b}$ with $b \approx-1$. The timedependence $x(t)$ can be extracted from $v(x)$ by integration. Because $x \sim t^{\alpha}$ is equivalent to $v \sim x^{1-1 / \alpha}, b=-1$ corresponds to a time dependence $x \sim t^{1 / 2}$ in good agreement with the theoretical prediction in Eq. (10).

Figure 7(b) shows the spreading velocity at fixed positions $x$ along the stripe as a function of stripe width. The strong dependence on stripe width - the experimental data indicate a power-law relation $v \sim w^{\chi}$ with $3.84 \lesssim \chi \lesssim 3.99-$ is also in excellent agreement with the prediction of Eq. (10). The size dependence indicates that the spreading process of the liquid rivulets is governed by the proposed hydrodynamic mechanism.

Figure 8 shows a comparison of the front shapes of a rivulet spreading in a $500-\mu \mathrm{m}$ wide channel when close to the inlet (left image) and close to the end (right image) of the channel. The difference in the spreading velocities is approximately a factor of 10 , yet the shape of the contact line is almost identical. Unfortunately, our optical interferometry setup cannot visualize the liquid-solid contact line directly. The first dark interference fringe occurs at a liquid thickness of $\lambda / 4 n$, where $\lambda$ is the wavelength of the light and $n$ the 

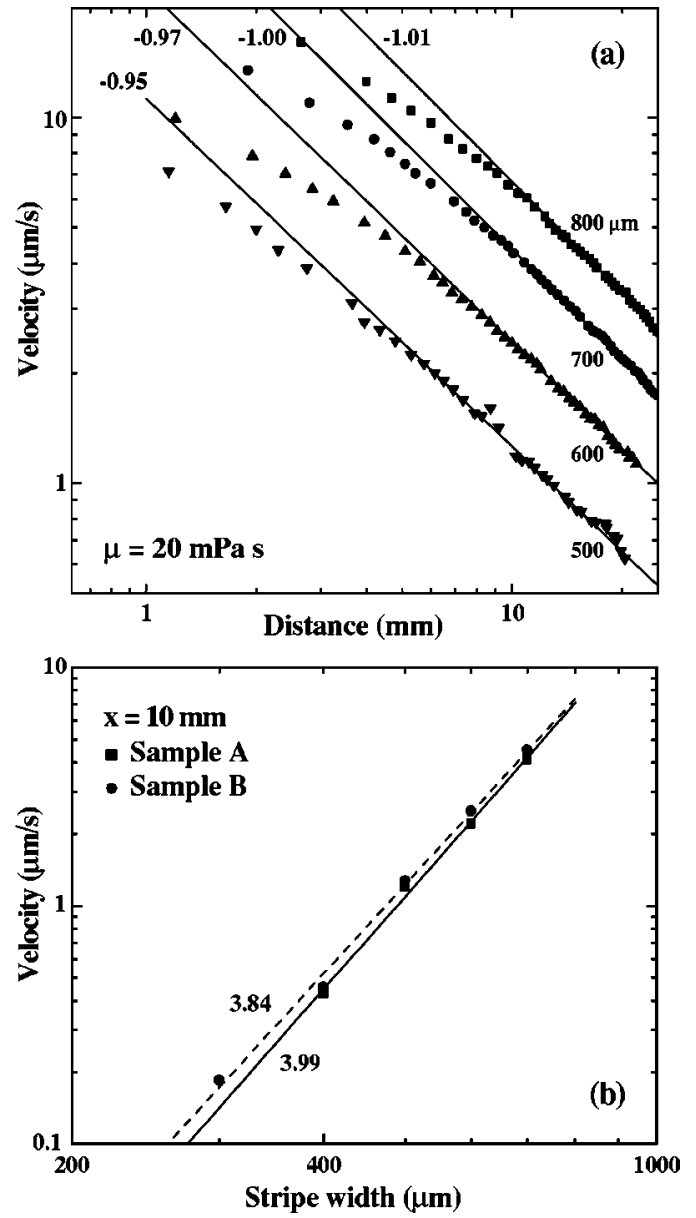

FIG. 7. (a) Experimental dependence of the spreading velocity $v$ on the distance from the reservoir inlet $x$. All measurements indicate a power-law behavior $v \sim x^{\beta}$ for $x$ exceeding several millimeters. The curves are labeled by the stripe width and the fitted exponent $\beta$. (b) Dependence of the velocity on the width of the channels for two different samples. The fitted exponents are 3.84 and 3.99.

refractive index of the liquid. The advancing contact anglereflected in the spacing of the interference fringes-is smaller in the right image, such that it appears shifted downwards relative to the left image. The continuous line superimposed on the images in Fig. 8 is a circular arc. Its shape is a good approximation to the contact lines except for the re-

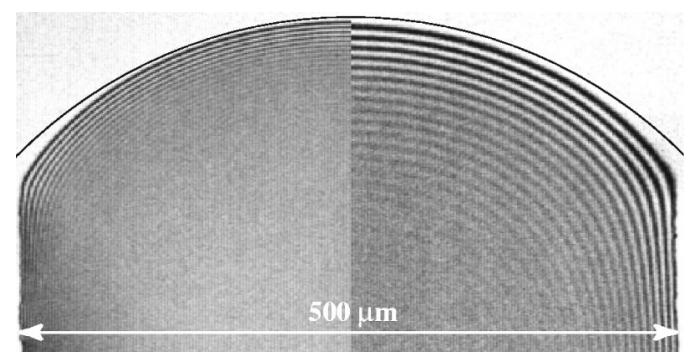

FIG. 8. Optical micrographs of the front region of a rivulet spreading in a $500-\mu \mathrm{m}$ wide channel. The left image was recorded close to the inlet, the right one close to the end of the stripe. The spreading speeds differed by approximately a factor of 10 . The continuous line represents an arc of a circle with radius $350 \mu \mathrm{m}$.

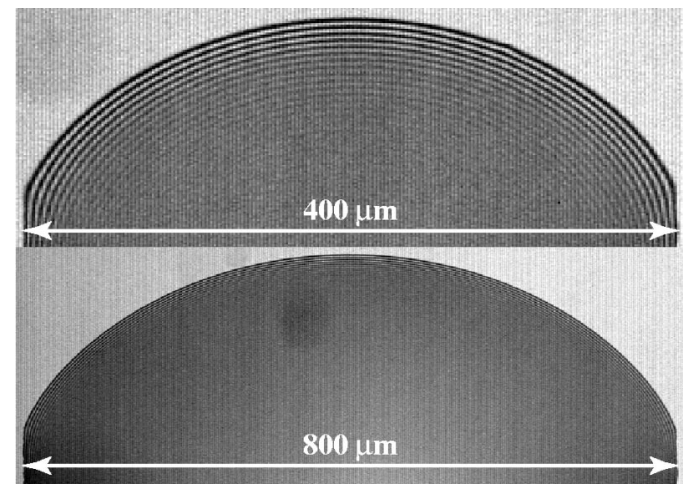

FIG. 9. Optical micrographs of the front region of rivulets spreading along a $400 \mu \mathrm{m}$ (top image) and a $800-\mu \mathrm{m}$ (bottom image) wide stripe.

gions close to the stripe edges. The radius of the circular arc is approximately $350 \mu \mathrm{m}$, i.e., larger than the value found for static rivulets.

Figure 9 shows the spreading front of two liquid rivulets on a 400- and 800- $\mu \mathrm{m}$ wide hydrophilic lane. From a comparison of the two images we deduce that the shape of the spreading contact line is practically identical despite the significant difference in the stripe width. Figures 8 and 9 reveal the velocity and size invariance of the contact line shape, which confirms that the liquid height profile is governed by the local capillary pressure in accordance with $\mathrm{Ca}$ and $\mathrm{Bo}$ being small for our system.

Figure 10 shows the spreading speed of a liquid rivulet at several distances from the inlet as a function of the volume deposited on the reservoir pad. The width of the channel was $700 \mu \mathrm{m}$. As can be seen, the velocity follows a power law $v \sim V^{\psi}$ with an exponent $\psi=2.65 \pm 0.1$, independent of the distance from the reservoir. This numerical value is again in good agreement with the theoretically predicted exponent of 2.76 .

\section{SUMMARY}

The spreading of a Newtonian liquid along hydrophilic microstripes exhibits the same $x \sim t^{1 / 2}$ dependence as a liquid

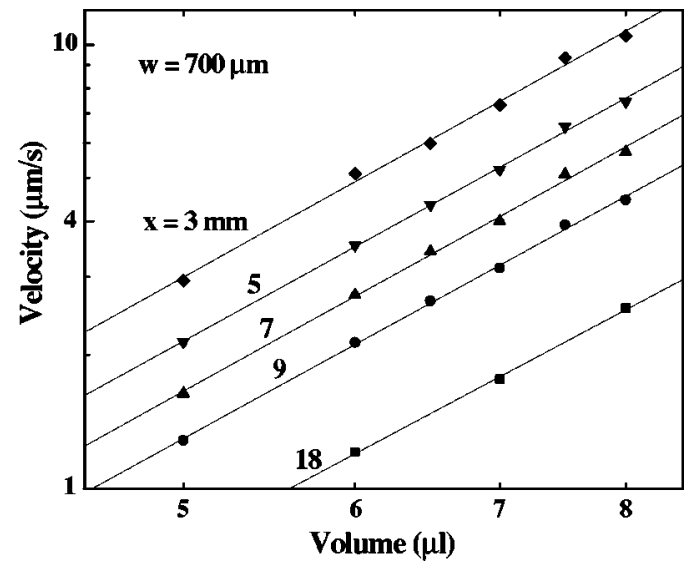

FIG. 10. Dependence of the spreading velocity on the volume of liquid deposited on the reservoir pad. The velocity was measured at distances of $3,5,7,9$, and $18 \mathrm{~mm}$ from the reservoir inlet on a $700-\mu \mathrm{m}$ wide stripe. 
spreading into a narrow groove despite the absence of capillary wicking. In analogy to wicking phenomena, however, the spreading kinetics is still governed by the same mechanism, namely, the existence of a constant pressure difference acting over an increasing distance. The predominance of the transverse curvature of the air-liquid interface increases the size dependence of the spreading velocity.

The influence of stripe edge roughness on the liquid profile critically depends on the roughness wavelength $\lambda$. High frequency variations, where $\lambda$ is smaller than the average channel width, are significantly damped, whereas long range fluctuations can induce noticeable liquid height variations. Small nonwetting defects on the hydrophilic lanes alter the height profile of the liquid over a length scale equal to the width of the channel, and do not significantly affect the $x$ $\sim t^{1 / 2}$ spreading dynamics in the limit of long distances.

\section{ACKNOWLEDGMENTS}

Financial sponsors of this work include the Princeton Center for Complex Materials (DMR-9809483), the NSF $X Y Z$ on a Chip program (CTS-0088774), the NSF POWRE Program (CTS-997353), and the DARPA-MTO Program on Molecular Level Printing. Discussions with Sigurd Wagner, Scott M. Miller, and Jeffrey M. Davis are gratefully acknowledged.
[1] P. G. de Gennes, Rev. Mod. Phys. 57, 827 (1985).

[2] E. B. Dussan, Ann. Rev. Fluid Mech. 11, 371 (1979).

[3] L. Leger and J. F. Joanny, Rep. Prog. Phys. 55, 431 (1992).

[4] L. H. Tanner, J. Phys. D 12, 1473 (1979).

[5] W. B. Hardy, Philos. Mag. 38, 49 (1919).

[6] F. Heslot, N. Fraysee, and A. M. Cazabat, Nature (London) 338, 640 (1989).

[7] M. P. Valignat, G. Oshanin, S. Villette, A. M. Cazabat, and M. Moreau, Phys. Rev. Lett. 80, 5377 (1998).

[8] B. D. Summ, A. V. Chadov, E. A. Raud, and Yu. V. Goryunov, Kolloidn. Zh. 42, 1010 (1980).

[9] E. A. Raud, Yu. V. Goryunov, B. D. Summ, and A. V. Chadov, Kolloidn. Zh. 47, 1200 (1985).

[10] Yu. V. Goryunov, E. A. Raud, B. D. Summ, and A. V. Chadov, Kolloidn. Zh. 47, 787 (1985).

[11] E. Raphael, J. Phys. (France) 50, 485 (1989).

[12] J. A. Mann, L. Romero, R. R. Rye, and F. G. Yost, Phys. Rev. E 52, 3967 (1995).

[13] R. R. Rye, J. A. Mann, and F. G. Yost, Langmuir 12, 555 (1996).

[14] R. R. Rye, F. G. Yost, and J. A. Mann, Langmuir 12, 4625 (1996).

[15] L. A. Romero and F. G. Yost, J. Fluid Mech. 322, 109 (1996).

[16] S. Gerdes, A.-M. Cazabat, and G. Ström, Langmuir 13, 7258 (1997).

[17] S. Gerdes, A.-M. Cazabat, G. Ström, and F. Tiberg, Langmuir 14, 7052 (1998).

[18] M. M. Weislogel and S. Lichter, J. Fluid Mech. 373, 349 (1998).

[19] F. M. Hosking, F. G. Yost, E. A. Holm, and J. R. Michael, J. Electron. Mater. 25, 1099 (1996).
[20] E. W. Washburn, Phys. Rev. 17, 273 (1921).

[21] U. Srinivasan, M. R. Houston, R. T. Howe, and R. Maboudian, IEEE J. Microelectro-mech. Syst. 7, 252 (1998).

[22] B. C. Bunker, R. W. Carpick, R. A. Assink, M. L. Thomas, M. G. Hankins, J. A. Voigt, D. Sipola, M. P. de Boer, and G. L. Gulley, Langmuir 16, 7742 (2000).

[23] V. V. Krotov and A. I. Rusanov, Physicochemical Hydrodynamics of Capillary Systems (Imperial College Press, London, 1999).

[24] P. Joos, P. van Remoortere, and M. Bracke, J. Colloid Interface Sci. 136, 189 (1990).

[25] L. R. Fisher and P. Lark, J. Colloid Interface Sci. 69, 486 (1979).

[26] K. Brakke, Exp. Math. 1, 141 (1992).

[27] M. M. Denn, Process Fluid Mechanics (Prentice Hall, Englewood Cliffs, NJ, 1980).

[28] L. Boltzmann, Ann. Phys. (Leipzig) 53, 959 (1894).

[29] J. Crank, The Mathematics of Diffusion (Oxford University Press, London, 1956).

[30] J. F. Joanny and P. G. de Gennes, J. Chem. Phys. 81, 552 (1984).

[31] J. F. Joanny and M. O. Robbins, J. Chem. Phys. 92, 3206 (1990).

[32] A. Paterson and M. Fermigier, Phys. Fluids 9, 2210 (1997).

[33] A. Paterson, M. Fermigier, P. Jenffer, and L. Limat, Phys. Rev. E 51, 1291 (1995).

[34] G. D. Nadkarni and S. Garoff, Europhys. Lett. 20, 523 (1992).

[35] M. E. Shanahan, Adv. Colloid Interface Sci. 39, 35 (1992).

[36] L. W. Schwartz, Langmuir 14, 3440 (1998).

[37] A. A. Darhuber, S. M. Troian, S. M. Miller, and S. Wagner, J. Appl. Phys. 87, 7768 (2000). 